\title{
Tyrosinase-catalyzed melanin as a contrast agent for photoacoustic tomography
}

Arie Krumholz, Sarah Chavez, Junjie Yao, Timothy Fleming, William E. Gillanders, et al.

Arie Krumholz, Sarah Chavez, Junjie Yao, Timothy Fleming, William E. Gillanders, Lihong V. Wang, "Tyrosinase-catalyzed melanin as a contrast agent for photoacoustic tomography," Proc. SPIE 7899, Photons Plus Ultrasound: Imaging and Sensing 2011, 78991G (17 February 2011); doi: $10.1117 / 12.879110$

SPIE. Event: SPIE BiOS, 2011, San Francisco, California, United States 


\title{
Tyrosinase-catalyzed melanin as a contrast agent for photoacoustic tomography
}

\author{
Arie Krumholz ${ }^{a}$, Sarah Chavez ${ }^{b}$, Junjie Yao ${ }^{a}$, Timothy Fleming ${ }^{b}$, William E. Gillanders ${ }^{b}$ \\ Lihong V. Wang ${ }^{\mathrm{a}}$ \\ ${ }^{a}$ Optical Imaging Laboratory, Department of Biomedical Engineering, Washington University in St. \\ Louis. One Boookings Drive, St. Louis, MO 63130 \\ ${ }^{b}$ Dept. of Surgery, Washington University in St. Louis. School of Medicine, 660 S. Euclid Ave., St. \\ Louis, MO 63110
}

\begin{abstract}
It is difficult to distinguish between tumor cells and surrounding cells without staining as is done in histology. We developed tyrosinase-catalyzed melanin as a reporter gene for photoacoustic tomography. Tyrosinase is the primary enzyme responsible for the production of melanin and alone is sufficient to produce melanin in non-melanogenic cells. Two cell lines were created: a stably transfected HeLa line and a transiently transfected 293 line. A phantom experiment was performed with the 293 transfected cells 48 hours post transfection and the results compared with oxygenated whole blood, B16 melanoma and 293 control cells. An in vivo experiment was performed using the transfected HeLa cells xenografted into a nude mouse ear, and then imaged. The results show strong contrast for tyrosinase-catalyzed melanin in both the 293 cells in the tube phantom as well as the in vivo result showing melanin in a nude mouse ear. Transfection increased expression in 293 cells 159 fold and image contrast compared to blood by as much as 50 fold. Due to the strong signal obtained at longer wavelengths and the decrease of blood signal at the same wavelengths, tyrosinase catalyzed melanin is a good candidate as a molecular imaging contrast agent for photoacoustic tomography.
\end{abstract}

Keywords: Tyrosinase, contrast agent, reporter gene, molecular imaging, photoacoustic tomography

\section{INTRODUCTION}

A reporter gene in the most basic sense is a gene product which can be measured and is linked to the product or function of interest [1]. One common reporter gene is $\beta$-galactosidase which was used previously by Li et al in 2007 [2]. In their experiment they showed the ability of PAM to image gene expression directly. When the lacZ gene is expressed, and X-gal (a substrate for $\beta$-galactosidase) is introduced, the enzyme cleaves X-gal produce a stable blue product. This blue product absorbs strongly in the red part of the visual spectrum so it is easily distinguishable from background hemoglobin absorption in vivo [2]. Razanski et al. have also shown that it is possible to image fluorescent reporter genes (eGFP and mCherry) in whole drosophila and zebra fish [3].

The OR-PAM system has been able to image single red blood cells (RBC) [4]. The main absorber in RBCs with a wavelength around $600 \mathrm{~nm}$ is hemoglobin. Hemoglobin has two states, oxy and deoxy with different absorption spectra that overlap at isosbestic points [5]. One of these isosbestic points is at $598 \mathrm{~nm}$ which is also near a peak for both forms [5]. At this wavelength the molar extinction coefficient of hemoglobin is $34,639 \mathrm{M}^{-1} \mathrm{~cm}^{-1}$, about two orders of magnitude greater than that of melanin. Melanin, as a single molecule is a not as strong an absorber as hemoglobin, however it is densely packed in cells and is therefore the absorption coefficient is comparable [6]. Hemoglobin is approximately $35 \%$ of the cell by volume, so in order to see a transfected cell expressing melanin with as strong a signal as is received from an RBC, then the melanin concentration in the cell must be around two orders of magnitude higher than that of hemoglobin. The signal from an RBC is strong, so although this volume would produce a similar signal, it is

Photons Plus Ultrasound: Imaging and Sensing 2011, edited by Alexander A. Oraevsky, Lihong V. Wang, Proc. of SPIE Vol. 7899, 78991G · C 2011 SPIE · CCC code: 1605-7422/11/\$18 · doi: 10.1117/12.879110 
possible to detect lower levels. The actual concentration of melanin in the cell has not been measured and is estimated from an average measure taken by Siegrist et al. [7].

Melanin production occurs primarily due to the tyrosinase enzyme. It does this through the conversion of tyrosine into DOPA and DOPA into dopaquinone, which then undergoes spontaneous cyclization and polymerization to create the final melanins [8]. When this enzyme is malfunctioning or absent albinism occurs [8]. Melanocytes and cells of this lineage are usually the only cells which can produce melanin; however Bouchard et al. showed in 1989 that transfecting a cell line, in their case fibroblasts, with tyrosinase cDNA was enough to produce melanin in nonmelanogenic cells [9]. The integration of the tyrosinase gene into normally non-melanogenic cells could prove to be a useful molecular imaging contrast agent for photoacoustic tomography.

\section{METHODS}

\subsection{Transfections}

The pReceiver-M02 plasmid vector was purchased from GeneCopoeia. Both transfections were performed with a modified Lipofectamine protocol. The transiently transfected 293 cells were used 48 hours post transfection. The stably transfected HeLa cells were transfected and placed under Geneticin selection for two weeks. The cells were kept under antibiotic selection until used.

\subsection{Preparation of sample: phantom}

4 Silastic ${ }^{\circledR}$ tubes of $300 \mu \mathrm{m}$ inner diameter were filled with varying samples as shown in table 1 . The tubes samples were first pelleted, then decanted and finally the tubes were filled using an insulin syringe.

Table 1: Phantom tube setup

\begin{tabular}{ll}
\hline \multicolumn{2}{l}{ Phantom } \\
\hline Tube & Cell type \\
1 & Oxygenated RBC \\
2 & B16 melanoma \\
3 & $293-w t$ \\
4 & $293-$ tyr \\
\hline
\end{tabular}

\subsection{Preparation of sample: in vivo}

A bolus injection of approximately $1 \mathrm{E} 6$, stably transfected HeLa cells, was xenografted into a nude mouse ear near a second order branching vessel. Imaging was performed one day after injection.

\subsection{Imaging}

The sample was imaged using two dimensional raster scanning on an OR-PAM system composed of a dye laser pumped by an Nd:YLF pump laser and detected with a $75 \mathrm{MHz}$ ultrasound transducer with a $70 \%$ nominal bandwidth as seen in figure 1. The prisms allows light to enter the sample and redirects the ultrasonic wave to the transducer [4]. The water 
tank is used for ultrasonic coupling between the sample and the transducer. The step size was $2.5 \mu \mathrm{m}$ and the scanning area varied between scans depending on the area of interest. For spectroscopic analysis 3 wavelengths were scanned consecutively: $584 \mathrm{~nm}, 590 \mathrm{~nm}$, and $600 \mathrm{~nm}$ for both the phantom and in vivo data.

\section{Light from dye laser}

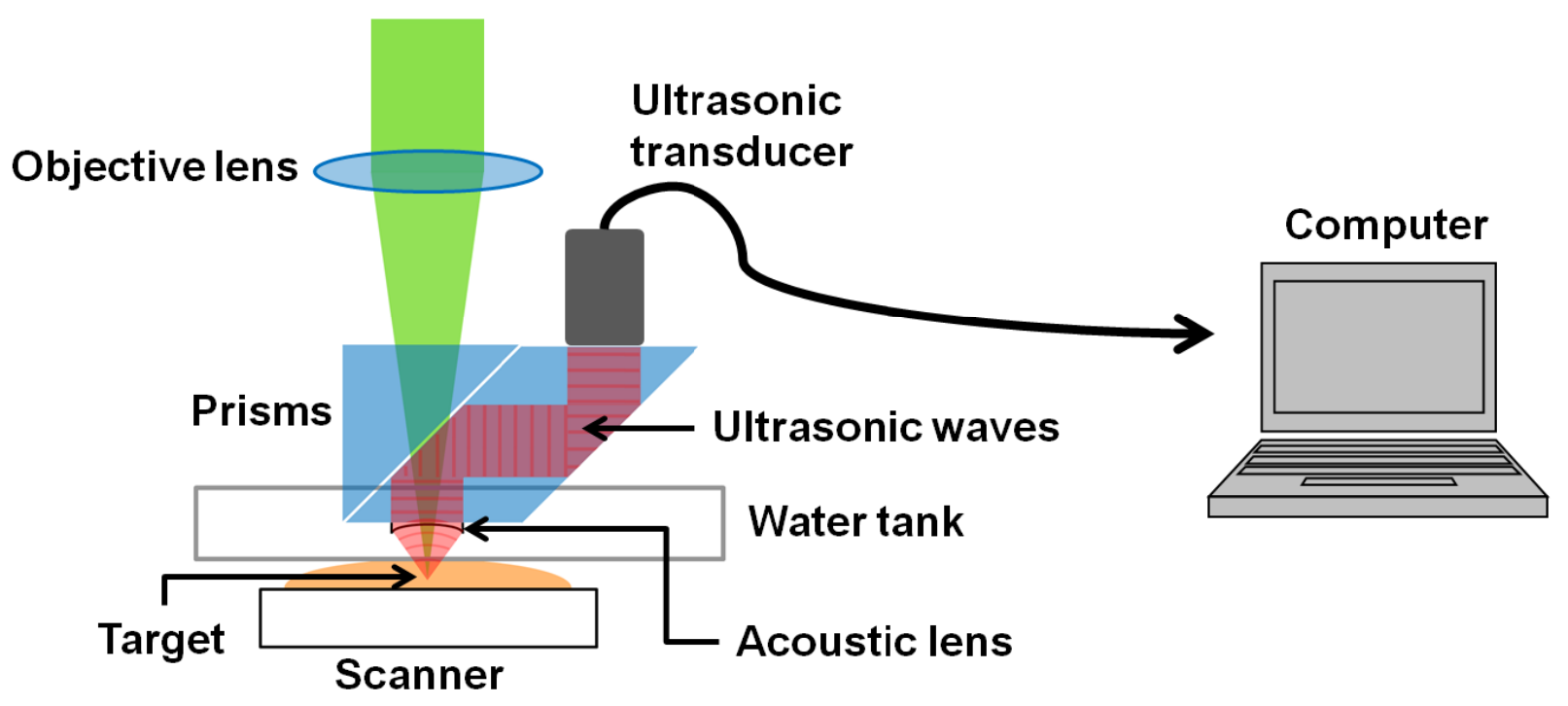

Figure 1: OR-PAM system used for imaging

\section{RESULTS}

\subsection{Phantom}

The images from the phantom were acquired using three wavelength measurements at $584 \mathrm{~nm}, 590 \mathrm{~nm}$ and $600 \mathrm{~nm}$. The four phantom tubes were glued parallel to each other onto a slide. The tubes were filled with the four samples: oxygenated whole blood in the top tube, B16 melanoma in the second, 293 control cells in the third tube and

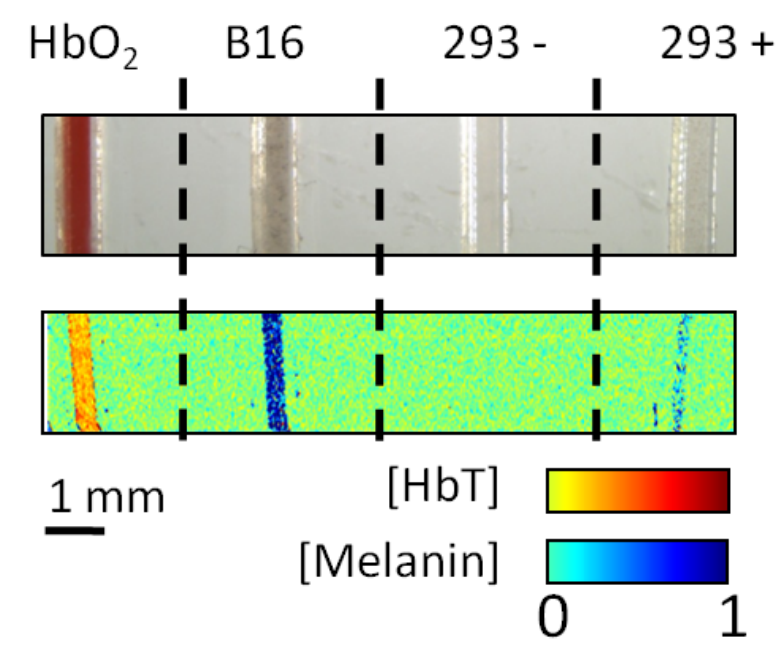
tyrosinase transfected 293 cells in the last tube. The cultured cells were first pelleted by centrifuge, the PBS decanted and then injected into each of the tubes independently with separate insulin syringes. The sample preparation is seen in figure 2 along with the maximum amplitude projection of the acquired photoacoustic signal showing normalized hemoglobin absorption in red and normalized melanin absorption in black. The images for both the phantom and in vivo results were acquired with about $100 \mathrm{~nJ}$ incident energy. As can be seen, the signal from blood, B16 melanoma and 293 transfected cells is strong while the signal from the control cells is small.

Figure 2: white light transmission photograph and PA MAP showing phantom setup and normalized absorption of blood and melanin. 
The signal from the tubes was quantified by taking the CNR of each tube as a function of the different wavelengths. As expected the signal from blood as for longer wavelengths decreases faster than the signal from melanin as the wavelengths progress. From the known molar extinction coefficients of both blood and melanin, it is known that the absorption for both decreases, however the slope of the decrease as wavelengths increase from $584 \mathrm{~nm}$ to $600 \mathrm{~nm}$ decreases more quickly for blood than for melanin. The CNR for the tubes follows the same trend with both blood signal and melanin signal decreasing as the wavelengths increase as can be seen in figure 3 . When taking the ratio between the CNRs of the melanin containing cells compared to the CNR of blood, the result should be a ratio of the contrasts for each of the absorbers. As can be seen in Figure $3 b$, it is clear that the signal from melanin increases compared to blood as the wavelength increases. Tyrosinase catalyzed melanin could be a good candidate for spectral separation in vivo.

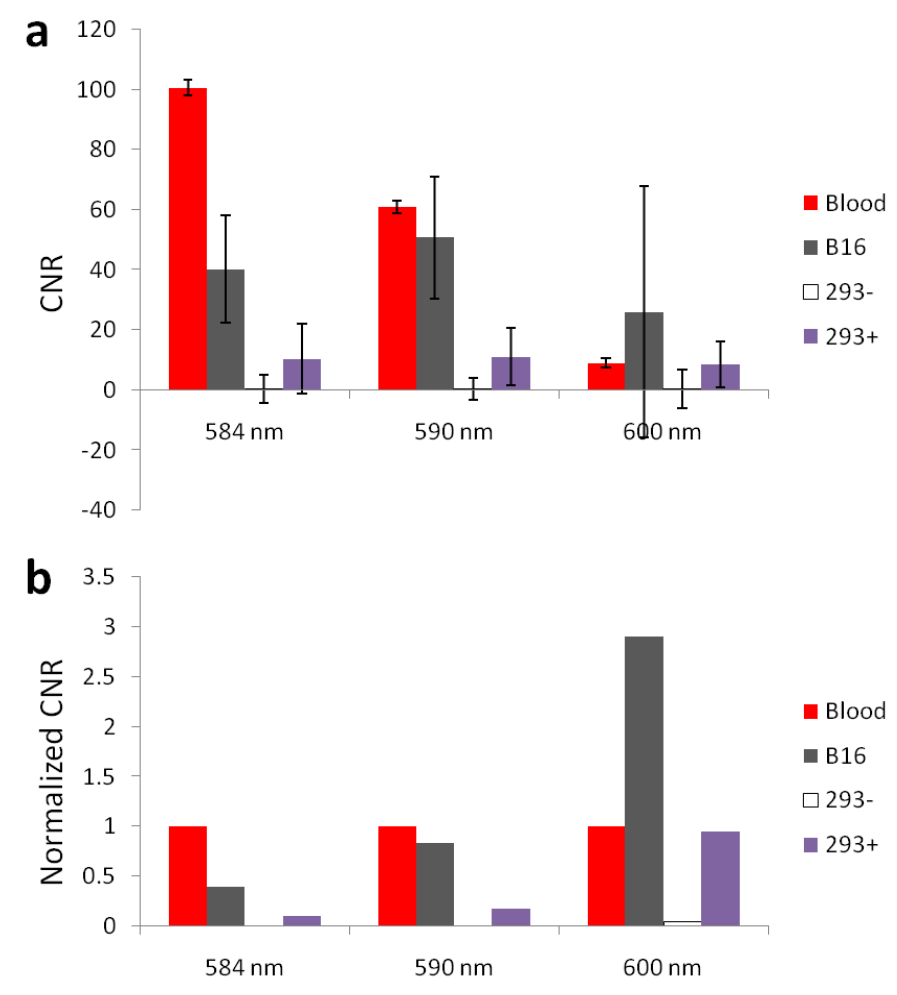

Figure 3: a) CNR of signal from tubes for oxygenated blood in red, B16 melanoma in dark grey, 293 control cells in white and 293 tyrosinase transfected cells in purple. b) Signal from the different tubes normalized by the blood signal

\subsection{In vivo}

The in vivo results were acquired using the same three wavelengths as the phantom image, $584 \mathrm{~nm}, 590 \mathrm{~nm}$, and 600 $\mathrm{nm}$. As can be seen from figure $4 \mathrm{a}$, there is some signal from melanin from the xenografted area shown in black. There is also a shadowing effect around the injection area due to an increase in the separation of the top and bottom layers of the ear. Due to this separation and the reduced Rayleigh length from the tight optical focusing, signals which are deeper are weak and not shown in the MAP. As can be seen in figure $4 \mathrm{~b}$, functional information can also be obtained from three wavelength measurements which are spectrally separated enough to allow for separation of the two main isoforms of hemoglobin: oxy-hemoglobin and deoxy-hemoglobin. 
a

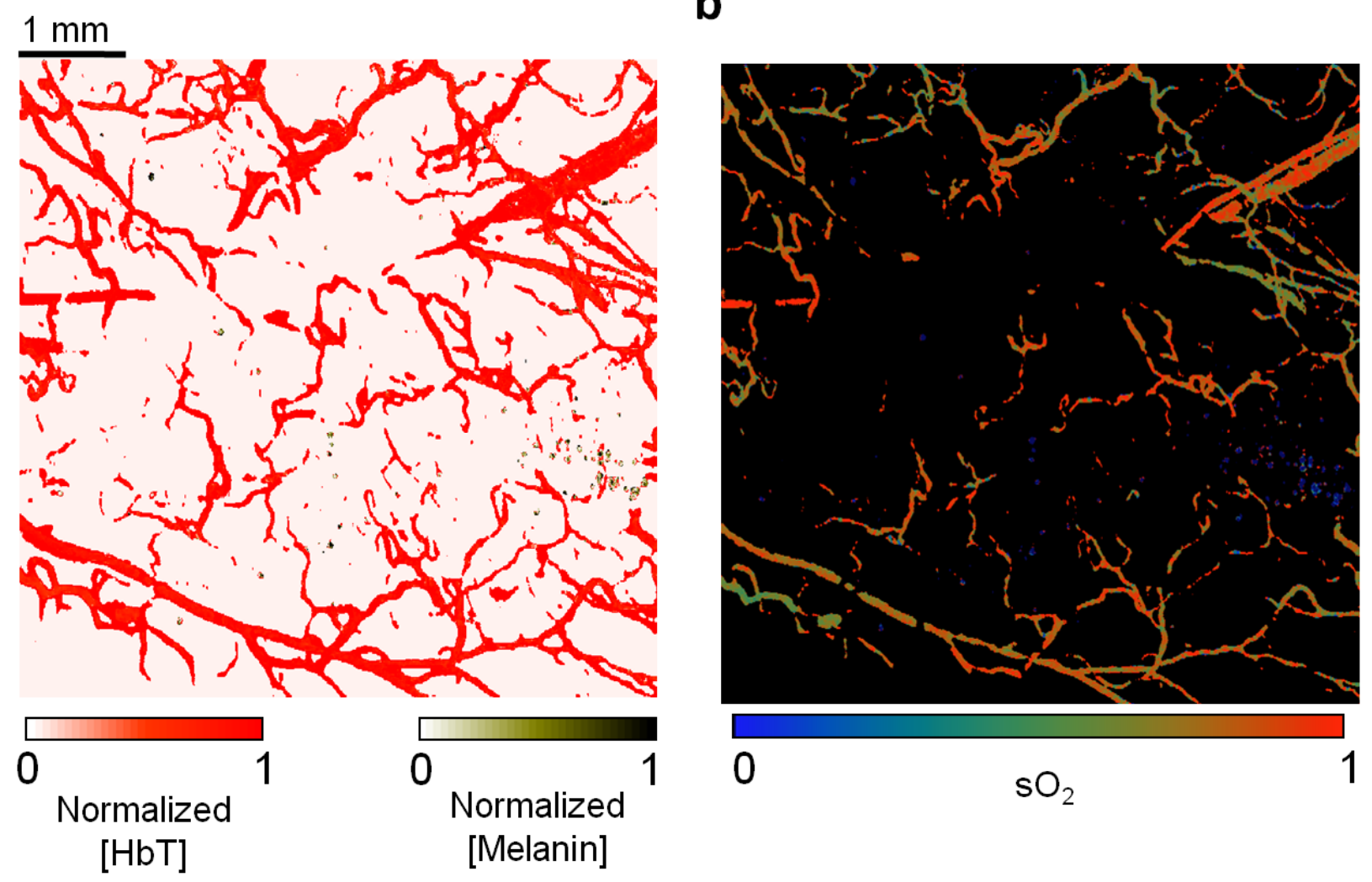

Figure 4: In vivo results for PA imaging of transfected HeLa cells

\section{SUMMARY}

It is difficult to distinguish clearly between tumor cells and the surrounding cells without histological staining. To increase the contrast of cells of interest such as tumor cells, a new contrast agent for photoacoustic tomography was developed by using melanin. Tyrosinase, the key enzyme in generating melanin in melanocytes, can be expressed in cells which normally do not produce melanin through the incorporation of the tyrosinase cDNA into the cells of interest. The expression of tyrosinase in non-melanogenic cells is sufficient to alone produce melanin. With the use of plasmid vectors it is possible to stably or transiently transfect the cells of interest and begin generating melanin for different applications. With a tissue specific promoter it might be possible to image disease states non-invasively and with high sensitivity. We have shown that it is possible to spectrally separate tyrosinase-catalyzed melanin from other absorbers in vivo and therefore have created a new molecular imaging contrast agent for photoacoustic tomography.

\section{REFERENCES}

[1] Markus S., Punch J., Lee W., "Motor- and Tail-Dependent Targeting of Dynein to Microtubule Plus Ends and the Cell Cortex," Current Biology, 19, pp. 196-205, 2009.

[2] Li L., Zemp R., Lungu G., Stoica G., Wang L., "Photoacoustic imaging of lacZ gene expression in vivo," JBO Letters, 12(2), pp. 02054-1-3, 2007.

[3] Ntziachristos V., Tung C., Bremer C., Weissleder R., "Fluorescence molecular tomography resolves protease activity in vivo," Nature Medicine, 8(7), pp. 752-760, 2002. 
[4] Maslov K., Zhang H., Hu Song., Wang L., "Optical-Resolution Confocal Photoacoustic Microscopy," SPIE proceedings, 2008.

[5] Wang, L.V, Wu, H., Biomedical Optics: Principles and Imaging (2007), Wiley, New Jersey, pp. 5, 115 165-171, 283312.

[6] Prahl S., "Optical Absorption of Hemoglobin," 15 Dec 1999, <http://omlc.ogi.edu/spectra/hemoglobin/index.html>, accessed 5 Aug 2009.

[7] Siegrist W., Eberle A., "In Situ Melanin Assay for MSH Using Mouse B16 Melanoma Cells in Culture," Analytical Biochemistry, 169, pp. 191-197, 1986.

[8] Tomita Y., Takeda A., Okinga S., Tagami H., Shibahara S., "Human oculocutaneous albinism caused by single base insertion in the tyrosinase gene," Biochemical and Biophysical Research Communications,164,3, pp. 990-996, 1989.

[9] Bouchard B., Fuller B., Vijayasaradhi S., Houghton A., "Induction of pigmentation in mouse fibroblasts by expression of human tyrosinase cDNA," Journal of Experimental Medicine, 169, pp. 2029-2042, 1989. 